\section{ARDS, up close and personal}

\author{
Mark Griffiths, ${ }^{1,2,3}$ Alastair Proudfoot ${ }^{4}$
}

The acute respiratory distress syndrome (ARDS) was first reported in a case series from Denver in $1967 .{ }^{1}$ Forty-five years later, the syndrome was reborn: the 'Berlin' definition captures patients with a mortality of $24 \%$ rising to $48 \%$ in the group of patients with the most severe respiratory failure. ${ }^{2}$ Survivors commonly suffer from muscle weakness and neuropsychiatric problems, such that fewer than $50 \%$ have returned to work 12 months after leaving intensive care. ${ }^{3}$ ARDS is important both clinically and financially. It is a not uncommon contributor to the deaths of critically ill patients of all ages ${ }^{4}$ and survivors commonly suffer from muscle weakness and neuro-psychiatric problems, such that fewer than 50\% have returned to work 12 months after leaving intensive care. $^{35}$

The onset of ARDS can be ascribed to two processes resulting from acute inflammation or injury at the lung's gas exchange surface: the alveolar-capillary membrane. These processes increase the permeability of the membrane associated with the recruitment of neutrophils into the airspace. The resulting acute inflammatory exudate inactivates surfactant, leading to collapse and consolidation with progressive loss of functioning lung. This would be well tolerated physiologically if the inflammatory process did not paralyse the lung's means of preventing ventilation-perfusion mismatch: hypoxic pulmonary vasoconstriction. The combination of these factors causes profound hypoxaemia accompanied by pulmonary oedema. The latter mechanism accounts for the fact that as a rule patients with ARDS are considerably more hypoxaemic than those with heart failure with similar radiographic appearances.

Since its recognition, much has been learnt about the epidemiology, clinical course and pathogenesis of ARDS, but these advances have failed to lead to a single effective 'disease modifying'

${ }^{1}$ Barts Heart Centre, St Bartholomew's Hospital, London, UK; ${ }^{2}$ National Heart \& Lung Institute, Imperial College, London, UK; ${ }^{3}$ William Harvey Research Institute, Queen Mary University of London, London, UK; ${ }^{4}$ Frederick Meijer Heart \& Vascular Institute, Spectrum Health, Grand Rapids, Michigan, USA Correspondence to Professor Mark Griffiths, Office 10 (BNB_01_411), 1st Floor—KGV Building, St Bartholomew's Hospital, West Smithfields, London EC1A 7BE, UK; m.griffiths@ic.ac.uk therapy. ${ }^{6}$ Is that such a surprise given that ARDS is not a disease but a label describing acute respiratory failure occurring de novo as a result of a plethora of pulmonary and non-pulmonary insults? Indeed, a common theme of research into ARDS and other critical illnesses has been the increasing appreciation of the contribution to the burden of ARDS of iatrogenic factors, most notably: fluid overload, ventilatorassociated lung injury from mechanical ventilation, transfusion of blood products and hospital-acquired infection (figure 1). ${ }^{7}$ While it is sobering to appreciate the negative role that well-intentioned healthcare systems have played, it has at least indicated the potential to prevent ARDS through simple quality-improvement interventions. ${ }^{89}$ ARDS investigators have been less successful in identifying patientspecific risk modifiers, although the Lung Injury Prediction Score (LIPS: table 1) represents the product of well-planned and well-executed epidemiology studies. ${ }^{10} \quad 11$ LIPS was designed to identify patients at high risk for ARDS prevention studies to be executed by the National Institutes of Health's Prevention and Early Treatment of Acute Lung Injury Network (http:/ petalnet.org/). The idea being that therapeutic interventions would be more likely to be effective if administered before the syndrome was fully established as has been the case in animal models. ${ }^{12}$ The Lung Injury Prevention with Aspirin Study (LIPS-A) is the first large multi-centre study using LIPS criteria to identify highrisk patients to address the question of whether ARDS can be prevented with a drug, in this case aspirin, ${ }^{13}$ the latest in a succession of promising therapeutics for ARDS which was supported by a plethora of positive preclinical data and basic science. Disappointingly, the study was negative and one contributing factor was that the score threshold for study inclusion produced only half the predicted number of ARDS cases, the study's primary outcome. This does raise concerns about the ability of LIPS to identify an enriched population of patients at risk of ARDS without the addition of factors such as biomarkers that can predict deterioration from at risk, to mild, to severe ARDS and to death.

An alternative strategy designed to increase the likelihood of positive clinical trials in ARDS is to select a less heterogeneous patient population-a step on the road to a personalised approach made at the expense of having a smaller pool of patients from which to recruit. Such splitting can be envisaged on the basis of readily identifiable predisposing causes (eg, influenza pneumonia, transfusion-related acute lung injury or systemic sepsis) or inherent patient characteristics, such as alcoholism or the expression of particular single-nucleotide polymorphisms known to be associated with a predisposition to

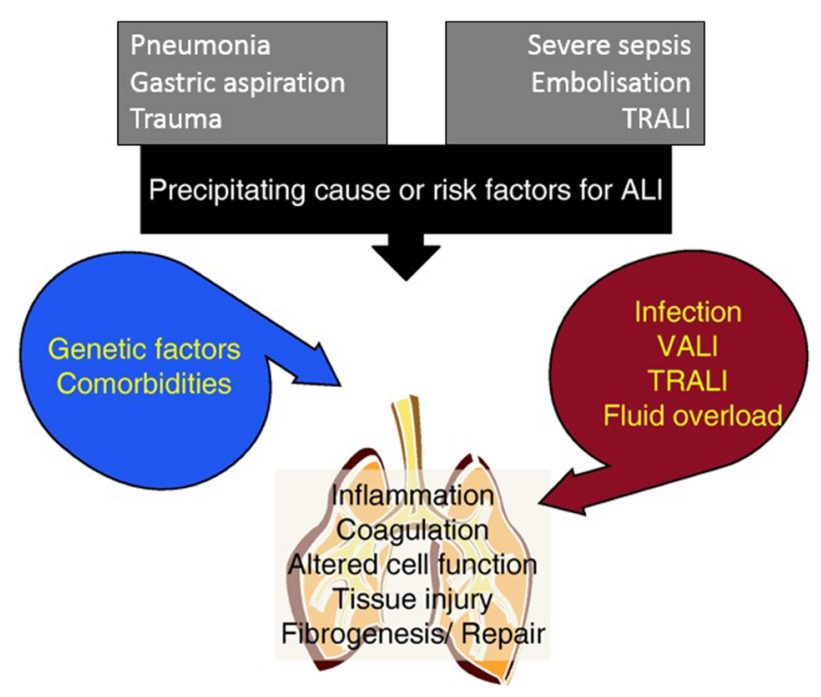

Figure 1 The evolution of ARDS. The pathogenesis of the syndrome depends on one or more initiating factors, which may affect the lung directly (eg, pneumonia) or via the circulation (eg, severe sepsis). These interact with patient-specific modulators (blue) and iatrogenic factors (red). The sum of a variety of pathological mechanisms manifests as ARDS. ARDS, acute respiratory distress syndrome; TRALI, transfusion-related acute lung injury; VALI, ventilator-associated lung injury. 
Table 1 The Lung Injury Prediction Score (LIPS)

\begin{tabular}{|c|c|c|c|}
\hline Predisposing conditions & LIPS points & Risk modifiers & LIPS points \\
\hline Shock & 2 & Alcohol abuse & 1 \\
\hline Aspiration & 2 & Obesity (BMI > 30) & 1 \\
\hline Sepsis & 1 & Hypoalbuminaemia & 1 \\
\hline Pneumonia & 1.5 & Diabetes mellitus & -1 \\
\hline High-risk surgery & & Chemotherapy & 1 \\
\hline Orthopaedic spine & 1 & $\mathrm{FiO}_{2}>0.35$ or $>4 \mathrm{~L} / \mathrm{min}$ & 2 \\
\hline Acute abdomen & 2 & & \\
\hline Cardiac & 2.5 & Tachypnoea RR >30 & 1.5 \\
\hline Aortic vascular & 3.5 & $\mathrm{SPO}_{2}<95 \%$ & 1 \\
\hline High-risk trauma & & Acidosis ( $\mathrm{pH}<7.35$ ) & 1.5 \\
\hline Traumatic brain injury & 2 & & \\
\hline Smoke inhalation & 2 & & \\
\hline Near drowning & 2 & & \\
\hline Lung contusion & 1.5 & & \\
\hline Multiple fractures & 1.5 & & \\
\hline \multicolumn{4}{|c|}{$\begin{array}{l}\text { LIPS discriminated between patients who did and who did not develop acute lung injury (ALI) when a cut-off score of } \\
>4 \text { points was used, sensitivity of the score for ALI was } 0.69 \text { ( } 95 \% \text { CI } 0.64 \text { to } 0.74) \text {, specificity } 0.78 \text { ( } 95 \% \mathrm{Cl} 0.77 \text { to } \\
0.79) \text {, positive predictive value } 0.18 \text { ( } 0.16 \text { to } 0.20) \text { and negative predictive value } 0.97 \text { (0.97 to } 0.98) \text {. Adapted from } \\
\text { Gajic, et al. }{ }^{10} \\
\text { BMI, body mass index. }\end{array}$} \\
\hline
\end{tabular}

ARDS, for example, the functional promoter variants in sphingosine 1-phosphate receptor $3 .{ }^{14}$ In this context, the work of Moazed et $a l^{15}$ is noteworthy and novel in that it aims to elucidate the mechanisms underlying the known predisposition of smokers to ARDS. The findings that indicate that smokers had an exaggerated response to bacterial endotoxin as measured by biomarkers of inflammation and increased alveolar-capillary membrane permeability are hardly surprising as these processes are interdependent and integral to the pathogenesis of ARDS. ${ }^{16}$ The additional suggestions that in smokers there is exaggerated epithelial injury and abnormalities of the vascular endothelial growth factor (VEGF) axis are hypothesis forming, but may suggest therapeutic targets that are more specific to this patient group. The pluripotent growth factor VEGF is critical for lung development and has multiple physiological roles subsequently depending on isotype and receptor interactions, including the regulation of vascular permeability and angiogenesis. ${ }^{17}$ Hence, it is tempting to speculate that smoking stresses the VEGF axis by 'demanding' continuously high alveolar repair activity which is underpinned by angiogenesis and that failure of this mechanism contributes to COPD. Similarly, VEGF could mediate some of the increased vascular permeability characteristics of ARDS and play a role in the later phases of recovery through repair by angiogenesis; consequently, the timing of any intervention targeting this axis would be crucial. to collaborate across continents in order to do this important translational work on humans rather than animal models. This effort reflects the widespread feeling that part of the failure of drug development has resulted from an overeagerness to jump from animal models into clinical trials where a clinical outcome is demanded. This increasing scepticism about animal models is due to fundamental interspecies differences in physiology and immunity, ${ }^{18}$ and their limited ability to reflect complex multifactorial human syndromes. ${ }^{19}$ This has particularly limited our understanding of injury and repair in the lung parenchyma and highlights the need for replacement of existing animal models of lung diseases with novel human models.

So what are the take home messages? Certainly, we need a heightened awareness of the ARDS diagnosis especially when there is an inclination to ascribe hypoxia in a smoker to mild COPD, thereby missing the opportunity to prevent the progression of the syndrome. A recent survey of 459 intensive care units in 50 countries from five continents, including 29144 patients, identified 3022 (10.4\%) cases fulfilling ARDS criteria, but in only half of the mild ARDS group was the syndrome recognised. ${ }^{20}$ Second, we need to continue to invest in human studies that aim to elucidate the pathogenesis of ARDS and to identify clinically useful biomarkers and surrogate outcome measures. ${ }^{21} 22$ Finally, these investigations need to be performed with a view to designing a stepwise
The authors have gone to great lengths approach to testing novel therapeutics in this particularly challenging patient group.

Twitter Follow Alastair Proudfoot at @ICUDocAP

Competing interests None declared.

Provenance and peer review Commissioned; internally peer reviewed.

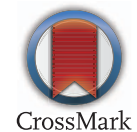

To cite Griffiths M, Proudfoot A. Thorax 2016;71:1073-1075.

Published Online First 20 September 2016

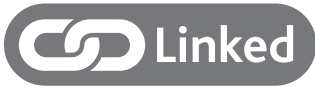

http://dx.doi.org/10.1136/thoraxjnl-2015-207886

Thorax 2016;71:1073-1075.

doi:10.1136/thoraxjnl-2016-208301

\section{REFERENCES}

1 Ashbaugh DG, Bigelow DB, Petty TL, et al. Acute respiratory distress in adults. Lancet 1967;2:319-23.

2 Ferguson ND, Fan E, Camporota L, et al. The Berlin definition of ARDS: an expanded rationale, justification, and supplementary material. Intensive Care Med 2012:38:1573-82.

3 Herridge MS, Cheung AM, Tansey CM, et al. One-year outcomes in survivors of the acute respiratory distress syndrome. $N$ Engl I Med 2003:348:683-93.

4 Rubenfeld GD, Caldwell E, Peabody E, et al. Incidence and outcomes of acute lung injury. $N$ Engl J Med 2005;353:1685-93.

5 Herridge MS, Tansey CM, Matté A, et al. Functional disability 5 years after acute respiratory distress syndrome. N Engl I Med 2011;364:1293-304.

6 Boyle AJ, Mac Sweeney R, McAuley DF. Pharmacological treatments in ARDS; a state-of-the-art update. BMC Med 2013;11:166

7 Salman D, Finney SJ, Griffiths MJ. Strategies to reduce ventilator-associated lung injury (VALI). Burns 2013:39:200-11.

8 Herasevich V, Tsapenko M, Kojicic M, et al. Limiting ventilator-induced lung injury through individual electronic medical record surveillance. Crit Care Med 2011;39:34-9

9 Pickering BW, Litell JM, Herasevich V, et al. Clinical review: the hospital of the future - building intelligent environments to facilitate safe and effective acute care delivery. Crit Care 2012;16:220.

10 Gajic O, Dabbagh 0, Park PK, et al. Early identification of patients at risk of acute lung injury: evaluation of lung injury prediction score in a multicenter cohort study. Am I Respir Crit Care Med 2011;183:462-70.

11 Trillo-Alvarez C, Cartin-Ceba R, Kor DJ, et al. Acute lung injury prediction score: derivation and validation in a population-based sample. Eur Respir J 2011;37:604-9.

12 Zarbock A, Singbartl K, Ley K. Complete reversal of acid-induced acute lung injury by blocking of platelet-neutrophil aggregation. I Clin Invest 2006;116:3211-19.

13 Kor DJ, Carter RE, Park PK, et al. Effect of Aspirin on Development of ARDS in At-Risk Patients Presenting to the Emergency Department: The LIPS-A Randomized Clinical Trial. JAMA 2016;315:2406-14. 
14 Sun X, Ma SF, Wade MS, et al. Functional promoter variants in sphingosine 1-phosphate receptor 3 associate with susceptibility to sepsis-associated acute respiratory distress syndrome. Am J Physiol Lung Cell Mol Physiol 2013;305:L467-77.

15 Moazed F, Burnham EL, Vandivier RW, et al. Cigarette smokers have exaggerated alveolar barrier disruption in response to lipopolysaccharide inhalation. Thorax 2016;71:1130-6.

16 Millar FR, Summers C, Griffiths MJ, et al. The pulmonary endothelium in acute respiratory distress syndrome: insights and therapeutic opportunities. Thorax 2016;71:462-73.

17 Papaioannou Al, Kostikas K, Kollia P, et al. Clinical implications for vascular endothelial growth factor in the lung: friend or foe? Respir Res 2006;7:128.

18 Seok J, Warren HS, Cuenca AG, et al. Genomic responses in mouse models poorly mimic human inflammatory diseases. Proc Natl Acad Sci USA 2013;110:3507-12.

19 Bastarache JA, Blackwell TS. Development of animal models for the acute respiratory distress syndrome. Dis Model Mech 2009;2:218-23.
20 Bellani G, Laffey JG, Pham T, et al. Epidemiology, Patterns of Care, and Mortality for Patients With Acute Respiratory Distress Syndrome in Intensive Care Units in 50 Countries. JAMA 2016;315:788-800.

21 Proudfoot AG, Hind M, Griffiths MJ. Biomarkers of acute lung injury: worth their salt? $B M C$ Med 2011;9:132.

22 Proudfoot AG, McAuley DF, Griffiths MJ, et al. Human models of acute lung injury. Dis Model Mech 2011;4:145-53. 